\title{
GASTBEITRAG
}

\section{Peer review revisited.}

Eine Untersuchung der SCM-Gutachten 2014/15

Peer review revisited.

An examination of the SCM reviews 2014/15

Michael Meyen \& Thomas Wiedemann 
Michael Meyen, Institut für Kommunikationswissenschaft und Medienforschung, LudwigMaximilians-Universität München, Oettingenstr. 67, 80538 München; Kontakt: michael. meyen(at)ifkw.Imu.de

Thomas Wiedemann, Institut für Kommunikationswissenschaft und Medienforschung, Ludwig-Maximilians-Universität München, Oettingenstr. 67, 80538 München; Kontakt: thomas.wiedemann(at)ifkw.Imu.de 


\title{
GASTBEITRAG
}

\section{Peer review revisited. \\ Eine Untersuchung der SCM-Gutachten 2014/15}

\section{Peer review revisited. \\ An examination of the SCM reviews 2014/15}

\author{
Michael Meyen \& Thomas Wiedemann
}

\begin{abstract}
Zusammenfassung: Der Beitrag beschäftigt sich mit dem Peer Review in der deutschsprachigen Kommunikationswissenschaft und fragt am Beispiel der Fachzeitschrift Studies in Communication I Media (SCM) nach der Rolle und der Funktion von Gutachten bei der Wissensproduktion. Ausgehend von der Skepsis, die dem Peer-Review-Verfahren bisweilen entgegengebracht wird, greift die Studie die wichtigsten Befunde zur Peer-Review-Forschung auf und konzeptualisiert die Gutachten als Kernelement der Kommunikation innerhalb der Fachgemeinschaft, in denen definiert wird, was heute gute Kommunikationswissenschaft ist. Die Untersuchung der 130 zwischen Mai 2014 und Dezember 2015 von SCM angeforderten Gutachten zeigt, dass sich rund anderthalb Jahrzehnte nach seiner Einführung längst ein Standard des Peer Review etabliert hat. Die Gutachter konstruieren sich dabei nach dem Prinzip der Kollegialität als Sympathisanten der Autoren und Anwälte der Fachgemeinschaft. Zugleich verbessern sie jeden der eingereichten Aufsätze und sind durch ihren Input sowie ihre Gatekeeper-Rolle entscheidend an der Produktion kommunikationswissenschaftlichen Wissens beteiligt. Peer Review reproduziert die Strukturen der Disziplin, weil das Verfahren die Mächtigen im Feld genauso stützt wie die Themen und Methoden, die am Machtpol für richtig und wichtig gehalten werden.
\end{abstract}

Schlagwörter: Kommunikationswissenschaft, Peer Review, Wissensproduktion, Selbstreflexion, Pierre Bourdieu

\begin{abstract}
This paper is about peer review in the German field of communication study. It focuses on the journal Studies in Communication I Media (SCM) and examines the role and function of reviews for the production of knowledge. Taking into account recent skepticism towards the peer review process, the study is based on the most important findings of the research on peer review and conceptualizes reviews as a core communication element within the field, which defines the dominant parameters of good communication research today. The analysis of 130 SCM reviews from May 2014 to December 2015 shows that 15 years after its beginning, peer review is an established process in the German speaking communication field. According to the principle of colleagueship, the reviewers construct themselves as sympathizers of the authors and advocates of the scientific community. At the same time, they improve every submitted paper and due to their input as well as their role as gatekeepers crucially influence the production of knowledge in German communication studies. However, peer review turns out to have an effect of reproducing the structures of the discipline, since this system supports the field's dominant
\end{abstract}


agents as well as the topics and methods, which are regarded as legitimate at the discipline's power pole.

Keywords: Communication study, peer review, knowledge production, self-reflection, Pierre Bourdieu

\section{Erkenntnisinteresse}

Dieser Beitrag beschäftigt sich mit dem Peer Review in der deutschsprachigen Kommunikationswissenschaft. Am Beispiel der Fachzeitschrift Studies in Communication | Media (SCM) wird nach der Rolle und nach der Funktion gefragt, die Gutachten von Kolleginnen und Kollegen bei der Wissensproduktion haben, und damit zugleich nach dem Entwicklungsstand der Disziplin. Definiert man Peer Review mit Peter Weingart (2001, S. 285) als „die Begutachtung und Bewertung“ von „wissenschaftlichen Wahrheitsbehauptungen durch die dazu allein kompetenten Kollegen“, dann entscheiden ausdifferenzierte Wissenschaften nicht „nach Parteibuch, Religionszugehörigkeit, massenmedialer Aufmerksamkeit oder Auflagenzahlen von Büchern “ über wissenschaftlichen Fortschritt, sondern ausschließlich nach „wissenschaftsimmanenten Gesichtspunkten“ (Gerhards, 2002, S. 19). Zugespitzt formuliert: Ohne Peer Review gibt es keine Wissenschaft. Der Soziologe Stefan Hirschauer (2004, S. 62-63) hat von einem „Kernstück wissenschaftlicher Kommunikation“ gesprochen, die Beurteilung von Manuskripten durch Fachzeitschriften dabei als „exemplarischen Fall aller Wissenschaftsevaluation“ bezeichnet und so auch die enorme Aufmerksamkeit erklärt, die die Forschung dem Peer Review widmet. Die Gutachten würden schließlich nicht nur dazu dienen, Herausgeberentscheidungen zu optimieren und zu legitimieren, sondern zugleich über die Reputation der Einreichenden entscheiden. Hirschauer (2004, S. 79-80) zufolge signalisieren „Publikationserfolge in begutachteten Zeitschriften“ neben „Schreibfleiß und Schreiblust“ vor allem, „dass jemand mit seinen Argumenten einige systematisch prüfende Leser in einer Konkurrenz mit anderen Autoren überzeugen konnte“. Deshalb handele es sich immer auch um „Rezeptionserfolge" (Hirschauer, 2004, S. 79).

In der deutschsprachigen Kommunikationswissenschaft ist Peer Review vergleichsweise neu. Wolfgang R. Langenbucher (2016), 1972 bis 2006 Mitherausgeber der Publizistik, hat das Auftauchen der Begriffe „Social Science Citation Index, Impact Factor und (Blind-) Peer Review“ auf „die Jahrtausendwende“ datiert, einen „totalen Bruch“ in der Zeitschriftenkultur der Fachgemeinschaft ausgemacht und mit Blick auf die Erfahrungen aus den letzten anderthalb Jahrzehnten sowie kritische Stimmen aus der Wissenschaftssoziologie eine „selbstbewusste Korrektur“ gefordert. Glaubt man Langenbucher (2016), dann hängt die Qualität einer Fachzeitschrift vor allem von den Beiträgern ab und nicht vom Begutachtungsverfahren. Während die Publizistik ab 2003 um anonymisierte Einreichungen bat, sich mit dem Start des Jahrgangs 2007 vom Herausgeberprinzip in Reinkultur verabschiedete und seitdem auch externe Gutachten heranzieht (vgl. Langenbucher, 2016), diskutierte die Mitgliederversammlung der Deutschen Gesellschaft für Publizistik- und Kommunikationswissenschaft (DGPuK) schon 
2001 in Münster über „eine von manchen als spektakulär empfundene Neuerung “. Die 48 Beiträge, die damals auf der Jahrestagung der Fachgesellschaft präsentiert wurden, waren erstmals in einem Review-Verfahren ausgewählt worden (Baum \& Schmidt, 2002, S. 11).

Langenbucher steht mit seiner Skepsis nicht allein. Im DGPuK-Informationsdienst Aviso warben Bernhard Debatin und Matthias Rath (2014/15) unlängst für die Aufhebung der Anonymität in Begutachtungsverfahren. In der von beiden betreuten „Debatte“ mit dem Titel „Die Blindheit der Gutachterei“ unterstützte zwar nur einer der sechs Beiträge diese Idee (Diana Marshall, eine Verlegerin und Zeitschriftenmanagerin aus der Biomedizin), auch die anderen Teilnehmer artikulierten aber Unbehagen. Helmut Scherer (2014/15) brachte drei „Bedenken“ auf den Punkt, die sich durch fast alle Statements ziehen: „Peer Review fördert den Mainstream, das wirklich Neue, das Originelle tut sich schwer. In einem kleinen Fach wie dem unseren ist Anonymität nur schwer zu wahren. Auch sind mitunter die Gutachten schlecht und wenig hilfreich. “ Melanie Magin und Sven Engesser (2014/15) wiesen außerdem auf die Auswahl der Gutachter hin („eine Vorentscheidung “), auf Qualitätskriterien, die instrumentell aktualisiert werden könnten, und auf Herausgeber, die sich manchmal über Bewertungen hinwegsetzen würden. Margreth Lünenborg (2014/15) und Friedrich Krotz (2014/15) wurden noch grundsätzlicher. Die Rede war hier von „subjektiven, fachfernen und interessengeleiteten Maßstäben“ (Lünenborg) sowie von einer „universellen Kontrollstrategie“ (Krotz), die schon deshalb auf die Inhalte des Fachs wirke, weil manche Publikationsformen leichter zu begutachten seien als andere (standardisiert vs. nicht standardisiert, empirisch vs. theoretisch, Hypothesentests vs. Theorieentwicklung).

Auf der gleichen Veranstaltung wie Wolfgang R. Langenbucher (2016) hat Thomas Hanitzsch (2016) in einem "Zwischenruf“ eine „Wissenschaftskultur“ beschrieben, in der „die genuin inhaltliche Rolle von Fachzeitschriften durch eine instrumentelle (Um-)Nutzung zunehmend an den Rand gedrängt wird“. Hanitzsch, der sich selbst als „Zeitschriftenenthusiast“ bezeichnet und von 2012 bis 2015 Herausgeber des renommierten ICA-Journals Communication Theory war, warnt vor „ernsten Konsequenzen für individuelle Wissenschaftlerkarrieren“ und „die disziplinäre Entwicklung des Fachs“, wenn die Zeitschriften nicht mehr nur „Taktgeber der Fachentwicklung und Instrument der wissenschaftlichen Qualitätssicherung“ sind, sondern „zunehmend“ zu dem Ort werden, an dem „Reputation und - mittelbar - auch Karrieren verhandelt werden“. Erstens seien „die Schwächen des Peer-Review-Systems . . . hinlänglich dokumentiert“, zweitens führe das Verfahren zum „Mainstreaming“ und zur „Normalisierung bestimmter wissenschaftlicher Perspektiven und Praktiken“, drittens leide die Qualität der Gutachten unter dem „geradezu wahnwitzigen Publikations-Wettrüsten“, und viertens stehe der „Publikations-Output“ in keinem Verhältnis zum Wissensgewinn, wenn nur noch „das so genannte Least Publishable Piece“ eingereicht werde - „Manuskripte, die vielleicht nicht sonderlich originell oder interessant sind, deren solide empirische Umsetzung es den Gutachtern aber schwer macht, sie aufgrund von methodischen Mängeln abzulehnen“. In einem ganz ähnlichen Abschiedsbeitrag als Herausgeber von Communication Theory hat Hanitzsch (2015, 
S. 353) außerdem darauf hingewiesen, dass Theorie-Aufsätze im Peer-ReviewSystem besonders riskant seien, da es hier höchst selten vorkomme, dass drei Gutachter übereinstimmen.

Die Beobachtungen der ehemaligen Zeitschriftenherausgeber Hanitzsch und Langenbucher sowie die Aviso-Debatte spiegeln die wichtigsten Befunde der PeerReview-Forschung. Kritisiert werden hier vor allem die geringe Übereinstimmung zwischen den Gutachtern, systematische Fehler bei der Bewertung (etwa: Status, Geschlecht, Alter oder persönliche Nähe und nicht wissenschaftliche Kriterien) sowie die geringe Prognosequalität für den Erfolg eines Beitrags (vgl. Ross, 1980; Ceci \& Peters, 1984; Cicchetti, 1991; Eysenck \& Eysenck, 1992; Wennerås \& Wold, 1997; Hirschauer, 2004; Bornmann, 2007). Stefan Hirschauer (2004, S. 73) hat auf die "Theorieschwäche" der entsprechenden Untersuchungen sowie ihre „normative Überdetermination“ hingewiesen („befangen zwischen Kritik und Apologetik“, teilweise „hoffnungslos evaluativ“) und vermutet, dass sich hier vor allem „enttäuschte Autoren“ am Gutachterwesen abarbeiten würden, während die Herausgeber das Verfahren eher verteidigen. So hat Richard Smith (2006) zwar kaum Unterschiede zu einem Münzwurf gesehen und sich daran erinnert, wie er als junger Redakteur des British Medical Journal fast einen Beitrag von Karl Popper abgelehnt hätte, ansonsten aber eine Parallele zur Demokratie gezogen: „a system full of problems but the least worst we have“ (Smith, 2006, S. 178). An diesem Argument sind bisher auch die meisten Vorschläge gescheitert, Peer Review über die technischen Möglichkeiten zu verbessern, die das Internet bietet (Stichworte: Kommentarfunktion, Commons, Weisheit der Vielen; vgl. Gould, 2012; Vesnic-Alujevic, 2012; Smith, 2014). Übersetzt man die Logik von Hirschauer in die Sprache von Bourdieu, dürften solche Vorschläge eher von Positionen kommen, die nicht zum Machtpol des wissenschaftlichen Feldes gehören und folglich ein Interesse daran haben, die Feldstrukturen zu ändern.

Die beiden wichtigsten Studien zum Peer Review in der Kommunikationswissenschaft suggerieren, dass man aus den Gutachten eine Art Rezept für einen erfolgreichen Aufsatz destillieren könne, und gehören damit in die Schublade „Amateur-Wissenschaftsforschung“ (Hirschauer, 2004, S. 73). Die Untersuchungskategorien wurden hier jeweils theoriefrei aus den Reviews selbst abgeleitet, ohne ihren Entstehungsprozess, die Sozialbeziehungen und die strategischen Überlegungen zu reflektieren, die in die Dokumente einfließen. W. Russell Neuman und Kollegen (2008) glauben so, auf der Basis von 117 Einreichungen für das Journal of Communication (davon 82 Ablehnungen) „sieben Todsünden“ und entsprechende „Tugenden“ nennen zu können. Karen Robson, Leyland Pitt und Douglas C. West (2015) sind für das Journal of Advertising Research ganz ähnlich vorgegangen (zehn Kriterien aus dem Material), haben aber neben Gutachten ( $n=$ 926 bei einer Ablehnungsquote von 85 Prozent) auch Interviews mit elf Mitgliedern des Editorial Board herangezogen. Das Erfolgsrezept ist in beiden Fällen nicht sonderlich überraschend: einen Beitrag zum Forschungsstand leisten, den theoretischen Rahmen definieren, klar schreiben und vor allem in den Methodenteil investieren.

Vor diesem Hintergrund werden hier 130 Reviews untersucht, die zwischen Mai 2014 und Dezember 2015 von der Fachzeitschrift Studies in Communication 
I Media angefordert wurden. Dabei geht es zwar auch um die Kriterien, auf die sich die Urteile jeweils stützen, um Empfehlungen für die Herausgeber und um den Grad an Übereinstimmung, wichtiger aber sind die Qualität der Gutachten, das Selbstverständnis der Reviewer und die Frage, welche Folgen das Peer Review für die deutschsprachige Kommunikationswissenschaft hat - sowohl inhaltlich als auch sozial. Die Gutachten werden dabei als ein Kernelement der Kommunikation innerhalb der Fachgemeinschaft konzeptualisiert: Hier wird definiert, was (im Moment) als ein guter wissenschaftlicher Aufsatz gilt und was als gute Kommunikationswissenschaft (Abschnitt 2). Dieser Ansatz wird durch die jüngste Mitgliederbefragung der DGPuK gestützt. „Gründliche und konstruktive Kritik zum Manuskript seitens der Reviewer“ wurde dort nach der „thematischen Ausrichtung der jeweiligen Fachzeitschrift" als wichtigster Grund für die Wahl eines Publikationsortes genannt (Echterbruch et al., 2016). Während Abschnitt 3 über das Untersuchungsmaterial und den Ablauf des Review-Verfahrens bei SCM informiert sowie aus der theoretischen Diskussion und den zur Verfügung stehenden Quellen die Forschungsfragen ableitet, die die Untersuchung leiten, fasst Abschnitt 4 die wichtigsten Ergebnisse in Thesenform zusammen und will so einen Beitrag leisten zu der „kritischen Auseinandersetzung “, die Wolfgang R. Langenbucher (2016) bei der Umstellung auf Peer Review vor anderthalb Jahrzehnten vermisst hat.

\section{Peer Review als „Sprechpraxis“ im wissenschaftlichen Feld}

Der Sozialpsychologe Lutz Bornmann (2007, S. 341) hat die „inkonsistenten Befunde“ der Forschung zum Peer Review auf die „theoretische Fragmentierung in der Wissenschaftssoziologie“ zurückgeführt. Bornmann unterscheidet dabei zwischen einer „Nordamerikanischen Schule“, die Peer Review als Garant dafür sieht, dass sich Wissenschaft an den Grundsätzen „guten“ wissenschaftlichen Handelns orientiert, und einer „sozialkonstruktivistischen Sicht“, die betont, dass wissenschaftliche Erkenntnis lokal und sozial konstruiert wird. Folgt man dieser Dichotomie, dann stützen sich Studien zum Peer Review vor allem auf zwei Normen aus dem Ethos der Wissenschaft: Universalismus und organisierter Skeptizismus (vgl. Merton, 1973). Gutachter seien einerseits dazu angehalten, „ausschließlich wissenschaftliche Gütekriterien und keine sachfremden Kriterien" anzulegen, „nur diejenigen Sachverhalte positiv“ zu beurteilen, „die mit den ,Gesetzen der Natur' übereinstimmen“, und so zu sichern, „dass Erkenntnisse unabhängig von lokalen und sozialen Bedingungen bei der Gewinnung (im Labor) oder Selektion (im Peer-Review) in der Wissenschaft akzeptiert und kommuniziert werden “. Andererseits garantiere die „kontroverse Diskussion unter Fachkollegen“, dass neues Wissen eingehend geprüft und dadurch auch legitimiert sei. Der Sozialkonstruktivismus dagegen lehnt schon die Idee ab, dass wissenschaftliches Handeln durch Normen wie Universalismus und organisierter Skeptizismus gesteuert wird, und kann dabei vor allem auf Studien zum Bias von Gutachtern verweisen (Bornmann, 2007, S. 332-338). So ist bekannt, dass die Publikationschancen sinken, wenn die Ergebnisse nicht signifikant sind, wenn sie den herrschenden Paradigmen oder der ,Blattlinie“ widersprechen und wenn es sich um Replikationen han- 
delt (vgl. Hirschauer, 2004, S. 64-65). Aus der Perspektive des Sozialkonstruktivismus hängt außerdem nicht nur der Forschungsprozess von den Bedingungen vor Ort ab, sondern auch das Peer Review. Die Biografie der Gutachterin, ihre „individuelle Interpretation des Fachwissens“, ihre Netzwerke und ihre Arbeitsbedingungen: All das fließt in den Text ein. Sozialkonstruktivisten erklären so die niedrigen Übereinstimmungsraten, die schärferen Urteile jüngerer Kolleginnen und Kollegen sowie die Annahme von Manuskripten, die von einer anderen Zeitschrift abgelehnt wurden, und sehen Veröffentlichungen außerdem als „ein gemeinsames Produkt von Autor, Gutachter und Herausgeber" und nicht als Eigentum derjenigen, die den Beitrag mit ihrem Namen zeichnen (Bornmann, 2007, S. 332-338).

Dieser letzte Punkt ist aus zwei Gründen wichtig. Zum einen entkräftet er die Befürchtung, die Gutachter könnten sich eine Idee aneignen, für eigene Publikationen nutzen und am Ende auch noch Urheberrechte reklamieren. Mindestens genauso plausibel ist die Annahme, dass Arbeit und Ideen von Gutachtern und Herausgebern in einen Aufsatz einfließen, ohne dass dieser Prozess für die Leser im Detail nachzuvollziehen ist. Stefan Hirschauer (2004, S. 74) hat deshalb vorgeschlagen, Publikationen als "hochartifizielle Individualisierungen kollektiver Forschungsprozesse“ zu betrachten - als „kommunikative Akte“, über die Wissenschaft ihre ,Individuen' erst herstelle. Zum anderen verweist die Idee des Gemeinschaftsprodukts auf die Position, die die Gutachten bei der Wissensproduktion haben. Bei SCM weiß die Reviewerin erstens, welchen Einfluss sie auf die Publikationsentscheidung und das Endprodukt hat, und zweitens, dass mindestens ein Redakteur ihre Identität kennt und vier Herausgeber sie wohlmöglich erahnen - Kolleginnen und Kollegen, die von der Mitgliederversammlung der DGPuK gewählt wurden und folglich hohes Ansehen genießen. Drittens muss die Reviewerin gerade in einer kleinen Fachgemeinschaft wie der deutschsprachigen Kommunikationswissenschaft immer fürchten, auch von den Autoren enttarnt zu werden - weil sie sich über Begriffe und Literaturhinweise verrät, weil man sich nach der Annahme des Aufsatzes gemeinsam freut oder weil sich die Gerüchteküche nicht um Anonymität schert.

Stefan Hirschauer $(2004$, S. 77) hat deshalb gefordert, Reviews als „Sprechpraxis“ zu konzeptualisieren - als „halböffentliche Sprechhandlungen“, die sehr viel verbindlicher sind als „das Geraune auf Tagungsfluren“. Was in einem Gutachten mitgeteilt werde, sei schon deshalb nicht mit dem zu verwechseln, was der Reviewer gedacht habe, weil Autoren, Herausgeber und Redakteure zum Kontext des Dokuments gehören. Dies gilt noch stärker, wenn (wie in dieser Studie) weder die verschiedenen Manuskriptversionen noch die Herausgebervoten oder die Kommunikation zwischen Herausgebern, Redaktion, Autoren und Gutachtern in die Untersuchung einbezogen werden können.

Dies führt direkt zum Ausgangspunkt des vorliegenden Beitrags: Die Gutachten für SCM geben Auskunft über den Stellenwert, den Peer Review in der deutschsprachigen Kommunikationswissenschaft hat, sowie über die Qualitätsstandards, die im Moment gelten. Dort wird gesagt, was die Gutachter mit einem reinen Gewissen von Autorinnen und Autoren einfordern zu können glauben, denen sie sich durch Kollegialität verpflichtet fühlen (vgl. Neidhardt, 1986). Mit 
Stefan Hirschauer (2004, S. 77) noch einmal anders gewendet: Die Argumente, die für die Annahme oder die Ablehnung eines Beitrags ins Feld geführt werden, und die Kriterien für die Beurteilung sind „immer auch rhetorische Ressourcen, also strategisch ,kontaminiert': Sie werden mobilisiert oder verschwiegen, mit Emphase versehen oder heruntergespielt." Dies erklärt, warum übereinstimmende Voten gar nicht so selten völlig unterschiedlich begründet und häufig Fehler in der Empirie moniert werden (vgl. Ceci \& Peters, 1983; Bakanic et al., 1989). Es kostet offenbar sehr viel weniger Aufwand, methodische Mängel zu beschreiben, als erkenntnistheoretisch zu argumentieren oder gar nachzuweisen, dass der Beitrag keinen Erkenntnisgewinn bringe (vgl. Hirschauer, 2004, S. 71). Damit sind zum einen die Grenzen von Review-Inhaltsanalysen angedeutet: Was sich im Text findet, legt die Gründe für ein Votum nur bedingt offen. Zum anderen macht es aus einer sozialkonstruktivistischen Perspektive wenig Sinn, mit den Kriterienkatalogen zu arbeiten, wie sie zum Beispiel Neuman et al. (2008) oder Robson et al. (2015) für Zeitschriften aus dem Gegenstandsbereich der Kommunikationswissenschaft verwendet haben. Diese Standards transportieren zum Teil „Zielkonflikte" (Hirschauer 2004: 77) und müssen nicht viel darüber sagen, warum ein Beitrag tatsächlich angenommen oder abgelehnt wurde.

Folgt man der Soziologie Pierre Bourdieus, dann ist das wissenschaftliche Feld eine soziale Welt mit Herrschaftsbeziehungen und Zwängen, zu der alle Akteure gehören, die Wissenschaft betreiben und die an die illusio des Feldes glauben, also an den Wert dessen, was dort auf dem Spiel steht. Auch wird hier davon ausgegangen, dass die Positionen einzelner Akteure sowie ihre Ressourcen von der Logik der Reputationszuweisung im Feld abhängen. Anders formuliert richtet sich die Struktur des Feldes bei Bourdieu nach der Verteilung des wissenschaftlichen Kapitals aus - der Anerkennung, die von der "Gesamtheit der gleich gesinnten Wettbewerber ... gewährt“ wird und die eine „besondere Art symbolischen Kapitals“ darstellt. Dass der Kredit eines Akteurs untrennbar mit den „objektiven Beziehungen“ im Wissenschaftsbetrieb verbunden und Ergebnis der Auseinandersetzungen zwischen herrschenden und beherrschenden Akteuren ist, leuchtet mit Blick auf das Peer-Review-Verfahren schlagartig ein. Denn dort kommt besonders zum Tragen, wie „die herrschenden Forscher oder Forschungen zu jedem Augenblick eine Gesamtheit der bedeutenden Gegenstände“ festlegen sowie die „Fragen, die für alle anderen Wissenschaftler Bedeutung haben, denen sie ihre Aufmerksamkeit widmen und deren Verfolgung sich schließlich ,bezahlt' macht" (Bourdieu, 1998, S. 18-23). Was in einer Disziplin also Relevanz besitzt und legitim ist, darüber entscheidet aus der Perspektive Bourdieus der Machtpol des Feldes. Stellvertretend für diesen dürften in der deutschsprachigen Kommunikationswissenschaft alle Kolleginnen und Kollegen stehen, die von den SCM-Herausgebern ausgewählt werden, um zu bestimmen, was gute Kommunikationswissenschaft ist und was nicht. Da diese Autorität jedoch stets umkämpft bleibt, ist es alles andere als verwunderlich, dass dem Peer Review unterstellt wird, interessengeleitet zu sein und den Mainstream zu fördern. Schließlich spiegelt sich darin das (aus Habitus und Position resultierende) Anliegen der herrschenden Akteure, die Feldstrukturen zu bewahren und denjenigen Kredit zu geben, die denselben Regeln wissenschaftlicher Praxis folgen. 


\section{Untersuchungsdesign}

Die vorliegende Studie stützt sich auf 130 Gutachten, die zwischen Mai 2014 und Dezember 2015 bei SCM eingegangen sind. Studies in Communication | Media erscheint seit 2011 als Online Journal der DGPuK, seit 2014 Open Access, und war die erste Fachzeitschrift der deutschsprachigen Kommunikationswissenschaft mit einem reinen Peer-Review-Verfahren. Zwar arbeiten auch die Publizistik (wie beschrieben) und Medien \& Kommunikationswissenschaft mit externen Gutachten, hier spielen aber nach wie vor Herausgeber bzw. Redaktion eine wichtige Rolle. Bei SCM wird ein anonymisiertes Manuskript an die Redaktion geschickt, die Titel und Abstract ohne Angabe der Verfasser an die vier Herausgeber weiterleitet. Dort werden thematisch und/oder methodisch passende Gutachter vorgeschlagen (in der Regel einschlägig ausgewiesene Professoren oder Postdocs). Die Redaktion prüft diese Vorschläge auf persönliche Nähe und Interessenskonflikte und bittet dann zwei potenzielle Gutachter um ein Review (Zeitfenster: drei Wochen). Für jede Zusage sind dabei im Durchschnitt 1,8 Anfragen nötig. SCM sieht für die Reviews kein standardisiertes Verfahren vor, schlägt als Anhaltspunkte aber die Kriterien Relevanz, theoretische Fundierung, Methode, Nachvollziehbarkeit und Klarheit der Darstellung vor. Ferner soll das Gutachten gegebenenfalls Überarbeitungsbedarf anzeigen sowie eine Einschätzung der Publikationswürdigkeit enthalten. Die Reviews werden den Herausgebern vorgelegt, die (ohne Kenntnis der Gutachter oder der Autoren) ein Votum über den Umgang mit dem Manuskript treffen. Diese Entscheidung wird den Autoren von der Redaktion mitgeteilt, verbunden mit einer Übersendung der anonymen Reviews. Ist keine unmittelbare Annahme oder Ablehnung erfolgt, haben die Verfasser nun die Möglichkeit, das Manuskript zu überarbeiten und zusammen mit einer Dokumentation der vorgenommenen Änderungen („Action Letter“) erneut der Redaktion vorzulegen, die das Paket dann an die Gutachter weiterleitet - die zweite Runde im Review-Prozess.

Nach Rücksprache mit den SCM-Herausgebern stellte Redakteur Andreas Scheu im Dezember 2015 drei Ordner mit 130 Reviews zur Verfügung, die seit Mai 2014 angefordert worden waren. Dort waren zwar auch die Titel der Einreichungen zu sehen, die Manuskripte selbst oder die Namen von Autoren bzw. Gutachtern wurden aber nicht übermittelt. Die Beschreibung des Materials in Tabelle 1 gibt keine Auskunft über die Annahmequote, da erstens ein Teil der Verfahren noch nicht abgeschlossen war (Ordner 3 ) und zweitens mindestens vier Manuskripte, die bereits abgelehnt worden waren (Ordner 1), offenbar erneut eingereicht wurden (dreimal mit Erfolg). Genauso wenig waren alle Reviews im Ordner 2 (,angenommen“) durchweg positiv oder führten direkt zur Veröffentlichung (jedenfalls nicht bis zum Februar 2016). 
Tabelle 1: Untersuchungsmaterial $(\mathrm{N}=130)$

\begin{tabular}{|l|l|}
\hline 1. Nicht oder nur nach umfassender Überarbeitung publikationswürdig: 38 Reviews \\
\hline Runde 1 & 34 Reviews (17 mal 2) \\
\hline Runde 2 & 4 Reviews (2 mal 2) \\
\hline 2. Sofort oder nach Überarbeitung publikationswürdig: 71 Reviews \\
\hline Runde 1 & 55 Reviews (27 mal 2,1 mal 1) \\
\hline Runde 2 & 14 Reviews (6 mal 2,2 mal 1) \\
\hline Runde 3 & 2 Reviews (1 mal 2) \\
\hline 3. Laufende Verfahren: 21 Reviews \\
\hline Runde 1 & 21 Reviews (9 mal 2,1 mal 3) \\
\hline
\end{tabular}

Um den Grad der Übereinstimmung zwischen den Gutachten in Runde 1 zu bestimmen, wurden vier Kategorien unterschieden: Annahme mit kleineren Änderungen, Annahme nach Überarbeitung, Ablehnung und Neueinreichung sowie Ablehnung. Lässt man die beiden Sonderfälle (nur ein oder drei Reviews) sowie die Gutachten ohne klares Votum beiseite, stimmten die Reviewer nur in jedem zweiten Fall überein (23 von 44 Fällen). Fünfmal war das Ergebnis sogar völlig konträr (Annahme vs. Ablehnung). Da die Kommunikation zwischen Herausgebern, Redaktion, Gutachtern und Autoren nicht untersucht wurde, da auch die Manuskripte selbst nicht zur Verfügung standen und da einige Manuskripte im Februar 2016 noch im Verfahren waren, kann über die endgültigen Entscheidungen nur spekuliert werden. Das gilt auch für die Frage, ob Lob und Kritik angemessen waren. Ohne die Manuskripte lässt sich nicht einmal mit Sicherheit sagen, dass sich die Reviews tatsächlich auf die Vorgehensweise und den Inhalt der begutachteten Beiträge beziehen.

Die Grenzen des Materials und die in Abschnitt 2 skizzierte theoretische Basis der vorliegenden Untersuchung erklären, warum es wenig Sinn gemacht hat, die Studien von Neuman et al. (2008) und Robson et al. (2015) für SCM zu replizieren und etwa über eine Regressionsanalyse die Faktoren zu ermitteln, die zur Annahme bzw. Ablehnung eines Beitrags führen. Stattdessen wurde ein qualitativer Zugriff gewählt. Dabei ging es zwar auch darum, die gegenwärtig im Fach dominierenden Kriterien für einen guten kommunikationswissenschaftlichen Aufsatz zu bestimmen, darüber hinaus jedoch ebenso um die Qualität der Reviews, um das Selbstverständnis am Machtpol des Feldes sowie um den Stellenwert des Peer Review für die Kommunikationswissenschaft im deutschsprachigen Raum. Aus der wissenschaftssoziologischen Forschung zum Peer Review, aus der theoretischen Perspektive und aus dem Untersuchungsmaterial ergaben sich folgende (sehr offene) Forschungsfragen:

- Welche Standards bedienen Reviews in der deutschsprachigen Kommunikationswissenschaft? Worüber wird in den Gutachten gesprochen und worüber vielleicht auch nicht? Anders formuliert: Ist der Vorwurf berechtigt, dass hier subjektiv, interessengeleitet und möglicherweise sogar unfair geurteilt wird? Finden sich im Untersuchungsmaterial Argumente für ein Open Peer Review?

- Wie konstruieren sich die Reviewer, wie legitimieren sie ihre Position, welches Wissenschafts-, welches Fach- und welches Selbstverständnis haben sie? 
- Welche Rolle spielt das Review-Verfahren in der Wissensproduktion des Fachs? Ist es für die Qualität der Fachzeitschriften tatsächlich egal, ob die Hefte von Herausgebern verantwortet werden oder über ein Review-Verfahren entstehen (vgl. Langenbucher, 2016)?

- Was gilt im Moment als gute Kommunikationswissenschaft und was bedeutet Peer Review für die Fachentwicklung? Gibt es Anzeichen für die „ernsten Konsequenzen“, vor denen Thomas Hanitzsch (2016) in seinem „Zwischenruf“ gewarnt hat?

Diese Forschungsfragen haben die Lektüre des Materials geleitet. Die 130 Reviews wurden zunächst von beiden Autoren unabhängig voneinander bearbeitet und codiert. Anschließend wurden die Zwischenergebnisse zusammengetragen, diskutiert und zu Befunden verdichtet (konsensuelles Codieren; vgl. Schmidt, 2008, S. 453).

\section{Ergebnisse}

Mithilfe von vier Thesen wird in diesem Abschnitt gezeigt, welche Rolle die Gutachten von Kolleginnen und Kollegen im Moment im Fach spielen. Der Aufbau folgt dabei den Forschungsfragen, die in Abschnitt 3 formuliert wurden. Die Thesen werden jeweils mit Ankerbeispielen aus dem Material illustriert und differenziert (nach dem Prinzip des ,pars pro toto'). Da die entsprechenden Zitate nicht belegt werden können, ohne die Zuordnung zu bestimmten Aufsätzen zu erlauben und damit die Vertraulichkeit zu brechen, die mit den Herausgebern von SCM vereinbart wurde, besteht selbstverständlich die Möglichkeit, das Untersuchungsmaterial bei den Autoren einzusehen.

These 1: Peer Review hat sich in der deutschsprachigen Kommunikationswissenschaft etabliert. Wer von SCM um ein Gutachten gebeten wird, weiß in aller Regel, was die Redaktion erwartet, obwohl keine formalen Vorgaben oder gar Beispiele mitgeliefert werden. Allerdings sind längst nicht alle Reviews, blind'. Da die Urheber des Aufsatzes gar nicht so selten direkt angesprochen werden, ist angesichts der Größe des Fachs anzunehmen, dass es hier eine Dunkelziffer gibt: Viele Reviewer ahnen oder wissen vermutlich, aus welcher Forschungsgruppe die Arbeit stammt.

Anderthalb Jahrzehnte nach dem „totalen Bruch“ in der Zeitschriftenkultur der Fachgemeinschaft (Langenbucher, 2016) gibt es einen Standard für die Begutachtung von Aufsätzen. Bei SCM haben die Reviews bei Erstbegutachtungen durchschnittlich eine Länge von rund 700 Wörtern (Mittelwert: 722, Median: 667; über 70 Prozent zwischen 400 und 1100 Wörtern bei $n=107$ ) und unterscheiden sich schon deshalb deutlich von Buchrezensionen, weil sie nicht den Inhalt des Beitrags zusammenfassen, sondern die „klassischen Kriterien“ abarbeiten, die Redakteur Andreas Scheu in seiner Anfrage nennt ( ,Relevanz, theoretische Fundierung, Methode, Nachvollziehbarkeit und Klarheit der Darstellung“"). 
Adressat sind in aller Regel die Herausgeber der Zeitschrift: Über die Autoren wird in der dritten Person gesprochen. Ausnahmen bestätigen diese Regel:

„Warum wollen Sie das eigentlich herausfinden? ... Haben Sie das mal faktorenanalytisch getestet? ... Der Leser erfährt nicht, wie die Bildung in die Regression aufgenommen wurde. War es 0, 1, 2 oder dummycodiert? Geben Sie das besser an. ... In der Conclusio fehlt mir ein Ausblick auf zukünftige Studien. Viel Erfolg mit dem Aufsatz!“

Die erste Abweichung von den international üblichen Standards betrifft die Publikationsempfehlung, die selbst bei viel Interpretationsfreude immer noch in acht Gutachten zu Ersteinreichungen vollkommen fehlt. Dies kann zwar am Untersuchungsmaterial liegen (keine E-Mails zwischen Reviewern, Redaktion und Herausgebern), dagegen spricht aber nicht nur das gerade zitierte Beispiel. In drei Gutachten gibt es statt einer Empfehlung eine Punkte-Tabelle, die an das Verfahren erinnert, das sich in der DGPuK für die Jahrestagungen der Fachgesellschaft und der Fachgruppen eingespielt hat. Bei vier anderen Aufsätzen haben die Gutachter einfach die Kommentarfunktion genutzt und ihre Anmerkungen direkt in das Dokument getippt, das zu bewerten war. In einem Extremfall hat es offenbar nicht einmal einen zweiten Lesedurchgang gegeben, da ein Kritikpunkt einige Absätze später lapidar zurückgenommen wird: „Okay, obiger Einwand im Grunde erledigt.“

Das Wissen um die Urheber des Textes wird immer dann offen gelegt, wenn es entsprechende Veröffentlichungen gibt - im Sample immerhin bei acht Aufsätzen. Ein Beispiel: „Der eingereichte Beitrag ist offenbar Teil eines Projekts, von dem ein anderer Teil 2013 bereits in der Publizistik erschienen ist. So ist es nicht möglich, hier ein ,blind Review“ abzugeben.“ Der Vorgängertext sei aber „im Literaturverzeichnis nachgewiesen“ und werde „von den Autoren selbst hinsichtlich ihres Vorgehens als ,interessant" qualifiziert“. Für die Beurteilung des Verfahrens interessant und ein Argument gegen die Aufhebung der Anonymitäts-Illusion: Wenn Reviewer ihr Wissen um die Urheber lüften, sind sie für eine Veröffentlichung. Gegenbeispiele sind ein Plädoyer für eine Neueinreichung und ein Aufsatz, den SCM dann nach zwei Review-Runden publiziert hat. Nachdem das entsprechende Gutachten in der ersten Runde noch empfohlen hatte, „den Beitrag - zumindest in dieser Form - eher nicht anzunehmen “, und „dringend“ dazu riet, die Verknüpfung beider Texte deutlich zu machen sowie den „Mehrwert“ herauszuarbeiten, hieß es nach der Überarbeitung bei aller Skepsis („Fleißbildchensammeln“; „Wo liegt das Neue?“): „Insgesamt kann man den Beitrag mit leichten Modifikationen sicherlich annehmen." Extrembeispiel für die Aushöhlung des Peer Review in einer Fachgemeinschaft, in der jede jeden kennt, ist ein Gutachten, in dem gleich zu Beginn die „Provenienz“ der Autoren gelüftet wird und dann eine Eloge folgt: „Das Paper stammt von einem in der deutschen Publizistik- und Kommunikationswissenschaft herausragenden xy-Forscher.“ Die „sehr gründliche theoretische Untermauerung “ gehe deshalb selbstverständlich „weit“ über das hinaus, „was für eine Fundierung der vorgelegten empirischen Studie notwendig gewesen wäre“, und die „Methodik“ sei „aufwändig und in keinem Punkt zu 
beanstanden“. Empfehlung an die Herausgeber: „eine sehr hochwertige Einreichung, deren Publikationswürdigkeit" außer Frage stehe.

These 2: Wer ein Gutachten für SCM schreibt, konstruiert sich nach dem Prinzip der Kollegialität als Sympathisant der Autoren und als Anwältin der Fachgemeinschaft. Entweder wird der Status eines Ko-Experten reklamiert oder der eines Laien, der sich zwar im konkreten Forschungsfeld oder mit bestimmten Methoden nicht auskennt, aber in der Kommunikationswissenschaft. Die Rhetorik ist selbst dann grundsätzlich positiv, wenn der Text obne Wenn und Aber abgelehnt wird.

Im Sample gibt es nur ein Gutachten, in dem offen von Überforderung gesprochen wird: „Der Text bietet eine schwierige Lektüre. Ich fühle mich ehrlich gesagt nicht kompetent genug, inhaltlich im Einzelnen Stellung zu nehmen. "Begründet wird dies sowohl mit dem Theoriehorizont und der Tiefe des Textes („,der Autor streut Reflexionen auch über das Universitätssystem und den Kunstmarkt ein“) als auch mit der Darstellungsform („mathematischer Formalismus“, „komplex und abstrakt", „die graphischen Modelle und Schaubilder"). „Ich habe mich auch gefragt, wer in Deutschland das entsprechende Vorwissen hat. . . . Mir ist niemand eingefallen. " Plädiert wird hier trotzdem für eine Veröffentlichung: weil der Autor „bereits anderswo positiv evaluiert worden ist“ und weil „eine Wissenschaft auch ungewöhnlichen, etwas außerhalb des ,Mainstreams' liegenden “ Ansätzen Platz bieten sollte. Auf der anderen Seite des Spektrums ist ebenfalls nur ein einziges Gutachten überhaupt nicht bereit, sich auf die Einreichenden einzulassen - bei einem Versuch, die Literatur zu einem bestimmten Gegenstandsbereich zu systematisieren. Empfohlen wird hier, die „theoretischen Befunde mit empirischen Analysen zu prüfen“. Bei der „Adressierung der Forschungsfrage“ sei „eine empirische Herangehensweise gegenüber der theoretischen Analyse“ zu bevorzugen.

Bei allen anderen Ablehnungen wird textimmanent argumentiert und kein Zweifel an der Fragestellung zugelassen. Die beiden Gutachten zu einer Experimentalstudie etwa heben gleich zu Beginn hervor, dass es sich um ein „wichtiges“ bzw. „interessantes und kommunikationswissenschaftlich relevantes Thema“ handelt (auch „von großer praktischer Relevanz“), um dann eigentlich jedes Detail anzugreifen: die Aufarbeitung des Forschungsstandes (,wenig zielgerichtet“), die Theoriearbeit („an der Oberfläche“), Design („,nicht akzeptabel“, „äußerst fragwürdig“), Stimulus und Datenanalyse („,nicht einzusehen, warum hier eine explorative Faktorenanalyse gerechnet wurde“) sowie Formalia („zahlreiche Orthographie-Fehler und Formulierungsschwächen“). Das Fazit fällt trotz dieser Fundamentalkritik versöhnlich aus: Es sei „wichtig“, dieses relevante Thema „weiterhin“ zu erforschen. Für die These, dass die Gutachten eher wohlwollend und unterstützend angelegt sind, spricht auch die Länge von Verrissen. Warum sollte man sonst knapp 1000 Wörter schreiben und detaillierte Hinweise für das Studiendesign und die Interpretation geben, wenn man die Verknüpfung von zwei Konzepten in einem Aufsatz für „nicht zulässig“ hält? Mit dem Prinzip der Kollegialität und vielleicht auch mit dem Wissen um die Größe der Fachgemeinschaft 
lässt sich erklären, warum die Reviews durchweg sachlich bleiben und auf Zuspitzungen oder gar persönliche Angriffe verzichten. Zumindest auf der Textoberfläche ist nichts von den ,subjektiven, fachfernen und interessengeleiteten Maßstäben“ zu finden, von denen Margreth Lünenborg (2014/15) in der Aviso-Debatte gesprochen hat.

Der Status als Ko-Experte, der letztlich auch die Publikationsempfehlung legitimiert, wird in den Gutachten erstens über Literaturhinweise konstruiert, die die Kenntnisse im jeweiligen Forschungsfeld dokumentieren, zweitens über eine Einordung des Aufsatzes, seiner theoretischen Fundierung und des Untersuchungsdesigns und drittens über methodisches Detailwissen, das vor allem über die Auswertungsverfahren demonstriert wird (Datenanalyse, Statistik). Dieser Punkt wird gleich in den Thesen 3 und 4 vertieft, da er sowohl etwas über die Rolle der Reviews im Prozess der Wissensproduktion sagt als auch über das, was im Moment als gute Kommunikationswissenschaft gilt. Bereits an dieser Stelle ist aber wichtig, dass nicht alle Reviewer für sich reklamieren, die methodischen Feinheiten besser zu kennen als die Autoren. Legitimiert wird das Urteil in solchen Fällen über die Funktion, die die Gutachten für die Fachgemeinschaft haben: Hier muss sichergestellt werden, dass die Publikationen anschlussfähig sind. Bei einer Medienwirkungsstudie beispielsweise geben beide Gutachter zu, „aus der Perspektive eines Nichtfachmanns“ zu urteilen, und fordern, „die Vorgehensweise auch für Leute mit mittleren methodischen Kenntnissen nachvollziehbar zu machen " (Gutachten 1; Nr. 2 fast wortgleich). Bei einer anderen Studie beschreibt sich die Gutachterin oder der Gutachter zunächst selbst „als Laie in diesem Fachgebiet“, um dann sehr detailliert Schwächen im Untersuchungsdesign zu monieren und eine Publikation abzulehnen.

These 3: Die Reviews sind ein zentrales Element der fachinternen Diskussion und verbessern jeden der eingereichten Aufsätze. Die Gutachterinnen und Gutachter übernehmen nicht nur Lektoratsaufgaben (Rechtschreibung und Grammatik, Zitierweise, fehlende Belege, Gestaltung von Abbildungen), sondern liefern auch Ideen für die theoretische Fundierung der Studien, ihr Design, die Auswertung sowie die Interpretation und sind so schon durch ibre Gatekeeper-Rolle entscheidend an der Produktion kommunikationswissenschaftlichen Wissens beteiligt.

In diesem halböffentlichen Forum ist es auch erlaubt, die Ausrichtung des Fachs ganz generell zu kritisieren - eine Möglichkeit, die im Sample allerdings nur zweimal genutzt wurde. In der Tendenz ganz ähnlich wie bei Thomas Hanitzsch (2016) attackiert ein Gutachten die „Parzellierung der Forschung in möglichst viele kleine ,Stückchen“, die einzeln publiziert werden können“ („nicht unbedingt originell“). Der Reviewer oder die Reviewerin moniert hier, dass so „der Blick auf das Ganze verloren“ gehe, und nimmt „amüsiert zur Kenntnis, dass in dem vorliegenden Papier" die Beschränkung auf studentische Stichproben kritisiert werde, obwohl die Autoren aus der gleichen „Küche“ mit diesem Rezept gerade bei einer hochrangigen US-Zeitschrift „reüssiert haben“ (implizites Votum: Neueinreichung). Bei einem anderen Text, der sehr gelobt und nach „einer gründlichen Überarbeitung“ zur Publikation empfohlen wird („die Darstellung der empiri- 
schen Studie zeigt, dass die Autorschaft ihr Handwerk beherrscht"), wird ebenfalls die Ausrichtung der empirischen Forschung im Fach angegriffen. Es fehle häufig an „einer Reflexion der eigenen Bedeutung“, an „einer theoretischen Fundierung“ und an einer „Einbettung in den weiteren wissenschaftlichen Diskurs“. Den Autoren wird am Ende ein thematisch einschlägiger Grundsatz aus der Zeitschrift GAIA ans Herz gelegt.

Diese beiden Beispiele werden hier auch deshalb so ausführlich dokumentiert, weil sie zeigen, dass Peer Review in der deutschsprachigen Kommunikationswissenschaft zumindest in der Sprechpraxis wie ein idealtypisches Gespräch unter Gleichrangigen funktioniert oder wie ein (nicht öffentliches) Wiki, welches das Wissen der Fachgemeinschaft anzapft, um die Qualität der Aufsätze und damit der Forschung insgesamt zu verbessern. Die Gutachterinnen und Gutachter kommunizieren den Glauben an das, was die Disziplin umtreibt (illusio), arbeiten mit einer Rhetorik der Anerkennung („Dieses methodische Setting ist anspruchsvoll und aufwendig in der Datenerhebung und -analyse") und bringen eigene Ideen ein („Meines Erachtens ist folgender Prozess vorstellbar“). Peer Review dient folglich nicht nur der Qualitätssicherung, wofür neben vielen Literaturhinweisen auch die unzähligen redaktionellen Verbesserungsvorschläge stehen, die Zeichensetzung und Tippfehler, die Tabellenbeschriftung, Englisch und den Streit um die aktuellste APA-Version einschließen. Viele Gutachten gehen weit über solche Details hinaus und liefern Anregungen, die die Reichweite der Befunde, die Interpretation der Ergebnisse und in einigen Fällen die gesamte Studie verändert haben dürften. Gar nicht so wenige Gutachten kommunizieren dabei die Freude, an diesem Prozess beteiligt zu sein. So ist in einem Gutachten von einer „kleinen, feinen Studie“ die Rede, "theoretisch hervorragend hergeleitet, empirisch pragmatisch und dennoch angemessen umgesetzt" sowie "dazu noch sehr gut geschrieben". Unter der Überschrift „Zum Finetuning“ folgt dann eine Liste mit 21 Punkten (in Runde 2 aus der gleichen Feder immerhin noch elf).

These 4: Peer Review reproduziert die Feldstrukturen in der deutschsprachigen Kommunikationswissenschaft, weil der Anschluss an die fachinterne Diskussion erstens das zentrale Relevanzkriterium ist, weil die Gutachten zweitens die umfassende Aufarbeitung des Forschungsstandes, drittens einen Beitrag zu diesem Forschungsstand und viertens die Einhaltung der formalen und inhaltichen Standards einfordern, die sich im Fach durchgesetzt haben, und weil die Publikationschance fünftens ganz entscheidend davon abhängt, wie die Einreichenden mit der Gutachter-Kritik umgehen. Das wichtigste Qualitätsmerkmal für gute Kommunikationswissenschaft ist dabei im Moment die Beherrschung des methodischen Handwerkszeugs. Auf intersubjektive Nachvollziehbarkeit, auf das Untersuchungsdesign und ganz besonders auf datenanalytische Finesse wird in den Reviews großer Wert gelegt.

Dieser letzte Punkt wird schon bei einem Blick auf den Platz deutlich, den Methodenfragen in den Gutachten einnehmen. Selbst wenn Reviewer die „klassischen Kriterien“, die Andreas Scheu in seiner Anfrage nennt, für die Gliederung ihres Statements nutzen, wird bereits in der Rubrik „theoretische Fundierung“ 
häufig die empirische Umsetzung der Forschungsfrage diskutiert (etwa die Begründung von Hypothesen oder die Operationalisierung bestimmter Konstrukte). Übersetzt in Empfehlungen für Autoren ließe sich dieser Kern der allermeisten Gutachten so zusammenfassen: Definieren Sie ihre zentralen Begriffe, begründen und problematisieren Sie jede Ihrer Entscheidungen (Hypothesen und Forschungsfragen, Design, Stichprobe, Instrumente, Auswertung), gehen Sie dabei bitte auch auf mögliche Alternativen ein, dokumentieren Sie den Forschungsprozess gründlich (Stichwort Transparenz) und sprechen Sie alle Befunde an, die aus dem Rahmen des (im Fach) Erwartbaren fallen. Vermutlich müsste man ergänzen: Schreiben Sie auf keinen Fall einen Beitrag, der sich nicht auf eine empirische Studie stützt. Bei allen Aufsätzen, die die Literatur systematisieren oder gar einen eigenen theoretischen Vorschlag unterbreiten, gibt es mindestens ein negatives Votum. Das entspricht den Erfahrungen von Thomas Hanitzsch (2015) und lässt sich auch damit erklären, dass „der Gutachter-Konsens“ disziplinübergreifend „bei Methodenkriterien höher ist als bei anderen“ (Hirschauer, 2004, S. 71).

Bei einem Beitrag, der abgelehnt wurde, setzt die Kritik schon bei der Herleitung der Untersuchungskriterien ein. Gutachten 1 meint, es wäre „sinnvoller“ gewesen, hier mit dem „Verfahren der multidimensionalen Skalierung“ zu arbeiten. Weiter im Text:

„Die Stichprobe ist ... extrem klein. Auch die Darstellung der Vorgehensweise finde ich nicht gut gelungen.... Auch erschließt sich mir die Entscheidung für eine Auswertung der Daten mittels Clusteranalyse nicht (es mag einen guten Grund geben, aber er wird nicht genannt). Zumindest hätten andere, für solche Studien gängigere Methoden und ihre Nachteile für den spezifischen Fall diskutiert werden können (etwa die Auswertung mit Q-Sort). Die konkrete Durchführung der Clusteranalyse könnte ebenfalls transparenter erläutert werden: Wie wurde die Zuordnung konkret gemessen, welche Daten gingen in die Analyse ein und wie wurden sie standardisiert? Was sind die aktiven Variablen?"

Gutachten 2 argumentiert ganz ähnlich und schlägt außerdem „die Durchführung einer Konfigurationsanalyse“ vor. Um nur noch aus einem zweiten Verfahren mit einem positiven Votum zu zitieren („, bei Berücksichtigung der Vorschläge geeignet zur Veröffentlichung“): „Aus methodischer Sicht fehlen Informationen dazu, in welcher Reihenfolge die Konstrukte/Items im Fragebogen abgefragt wurden. “ Die Gutachterin oder der Gutachter schlägt außerdem vor, „eine ungerichtete Korrelation“ zu berechnen (anstelle eines Pfadmodells), kritisiert einen „Messindikator“ („uneindeutig“) und vermisst einige „statistische Angaben“.

Neben dem methodischen Vorgehen gehört auch der Aufbau eines Aufsatzes zu den Standards, die im Peer Review verteidigt werden. Wer von der klassischen Reihenfolge (Erkenntnisinteresse, Theorie und Forschungsstand, Untersuchungsdesign, Ergebnisse, Diskussion und Ausblick) abweicht oder gar Teile weglässt, hat keine Chance, das Verfahren erfolgreich zu überstehen. Das Gleiche gilt für Texte, die das, was die Fachgemeinschaft über Forschung zu wissen glaubt, ignorieren. Bei einer Studie, die sich vor allem auf einige wenige Leitfadeninterviews stützt und nach zwei Review-Runden veröffentlicht wurde, forderten beide Gutachter nicht nur, die Struktur zu ändern („,verwirrend“; „Die theoretische Grundlage des Beitrags wird - ungewöhnlicher Weise - an zwei verschiedenen Stellen 
dargelegt"), sondern kritisierten unisono die Prämissen des Autors. Zum einen sei die dargelegte Auffassung von Triangulation „merkwürdig“ (,irreführend“), und zum anderen könne man mit dem (qualitativ erhobenen) Material grundsätzlich nicht zu „generalisierbaren Aussagen“ kommen. „Auch dass teilnehmende Beobachtungen immer als Einzelfallstudien konzipiert sein sollen, kann ich nicht nachvollziehen." Im zweiten Durchgang loben beide Gutachter, dass der Autor auf ihre „Anregungen“ eingegangen ist. „Schon das rechtfertigt es, nun zu veröffentlichen. "Nur wer die Deutungsmacht der Fachgemeinschaft (hier vertreten durch die Reviewer von SCM) anerkennt, wird als Diskursteilnehmer zugelassen.

Solche Abweichungen von ,normaler' Kommunikationswissenschaft sind allerdings selten. Nur in jeweils zwei Verfahren wurden der Aufbau der Aufsätze oder erkenntnistheoretische Fragen thematisiert. Da die Reviewer selbst zum Feld gehören, dieses Feld für wichtig halten (illusio) und neben den generellen methodischen Standards auch die Literatur in einem bestimmten Forschungsgebiet sehr gut kennen, hängt die Veröffentlichung eines Aufsatzes vor allem davon ab, ob er einen Beitrag zum Forschungsstand leistet. Das heißt auch: Wenn sich ein bestimmtes Thema (möglicherweise sogar mit einer bestimmten Herangehensweise) einmal im Kanon der Fachgemeinschaft etabliert hat, dann wird nicht mehr gefragt, ob Thema und Herangehensweise sinnvoll oder gar jenseits der Fachgrenzen relevant sind. In der „Sprechpraxis“ des Peer Review wird zwar gar nicht so selten auch auf die Gesellschaft und die Berufsfelder verwiesen, die sich im Themenspektrum der Kommunikationswissenschaft entwickelt haben, die Rekrutierung der Gutachter (ausgewiesen durch Forschung in einem bestimmten Gebiet), ihr Selbstverständnis (in der Regel: Ko-Experte), der Kriterienkatalog von SCM sowie die Fixierung auf Untersuchungsdesign und Datenanalyse führen aber dazu, dass die Bewertung letztlich ausschließlich von wissenschaftsinternen Maßstäben abhängt. Wieder in Empfehlungen für Autoren übersetzt: Arbeiten Sie den Forschungsstand gründlich auf, zitieren Sie die Autoren, die als zentral gelten (schon weil es sich um potenzielle Gutachter handelt), und machen Sie deutlich, welchen (kleinen) Beitrag Sie leisten. In einem Extrembeispiel arbeitet sich ein Gutachter in zwei Runden an einem Aufsatz ab, der die Rezeption seines eigenen Ansatzes zunächst unterschätzt und ihn auch nach der Überarbeitung „nur strategisch “ zitiert, aber weder „voll und ganz“ noch „fair“. „Sauber gegeneinander gestellt mit allen Faktoren. Gern auch in Form einer Abbildung. Und dann (und nur dann) kann sich der Leser des Artikels selbst eine Meinung bilden. Einen solchen Artikel würde ich gern lesen. Das ist aber nicht (oder: noch nicht) der Artikel, der hier zum Review vorlag.“

\section{Ausblick}

Die deutschsprachige Kommunikationswissenschaft sollte der Versuchung widerstehen, bei ihren Zeitschriften zum Herausgeberprinzip zurückzukehren. Ein solcher Schritt wäre keine „selbstbewusste Korrektur“ (Langenbucher, 2016), sondern Selbstverstümmelung. Die Gutachten, die 2014/15 für SCM geschrieben wurden, bringen eine solche Fülle an Wissen in den fachlichen Diskurs ein, wie es einige wenige Herausgeber nie und nimmer haben können. Vielleicht war es 1972, 
als Wolfgang R. Langenbucher Mitherausgeber der Publizistik wurde und die Kommunikationswissenschaft in der Bundesrepublik Deutschland an nur sieben Standorten mit jeweils einer Professur vertreten war (vgl. Meyen, 2004), noch möglich, das Fach in all seinen Facetten zu überschauen und in jedem Forschungsgebiet auf dem Laufenden zu sein. In der sehr viel größeren und stark ausdifferenzierten Disziplin der Gegenwart kann dies niemand mehr leisten. Ganz unabhängig von der Literaturrezeption oder theoretischen und methodischen Fragen übernimmt die Fachgemeinschaft in Gestalt ihrer Reviewer Lektoratsaufgaben, entlastet dadurch die ehrenamtlichen Herausgeber und verbessert die formale Qualität der Beiträge weit stärker, als das ein einzelner Redakteur könnte.

Die vorliegende Studie liefert außerdem keine Argumente für ein Open Peer Review, wie es Bernhard Debatin und Matthias Rath (2014/15) im Aviso angeregt haben - eher im Gegenteil. Nahezu alle untersuchten Gutachten setzen sich ernsthaft und ausführlich, sachlich und fair mit den Ideen der Einreichenden auseinander und liefern selbst dann noch Anregungen und Vorschläge, wenn sie eine Veröffentlichung rundweg ablehnen. Das mag mit dem Prinzip der Kollegialität zu tun haben, mit der Größe einer Fachgemeinschaft, in der man sich kennt, oder mit dem Charakter der Reviews als "halböffentliche Sprechhandlungen “ (Hirschauer, 2004, S. 77), aber trotzdem: Nimmt man die (zugegeben wenigen) Fälle im Sample, in denen die Reviewer offenlegen, dass sie die Autoren kennen, wäre eher zu befürchten, dass eine Aufhebung der Anonymität die (bei SCM ohnehin vorhandene) Tendenz zu positiven Bewertungen verstärken würde.

Nicht von der Hand zu weisen sind dagegen die Einwände, die Thomas Hanitzsch (2016) in seinem „Zwischenruf“ formuliert hat. Peer Review ist nicht nur in jeder Hinsicht strukturkonservativ, weil das Verfahren die Mächtigen im Feld genauso stützt wie die Themen und Methoden, die am Machtpol für richtig und wichtig gehalten werden, sondern auch weitgehend blind für Fragen nach der gesellschaftlichen Relevanz. Den Forschungsstand ein Stück weiterbringen und dabei methodische Expertise beweisen: Das ist der Mechanismus, der zur Praxis des „Least Publishable Piece“ führt, zum „Mainstreaming“ und zum Verlust an Originalität (Hanitzsch, 2016). Helmut Scherer (2014/15) hat die Kolleginnen und Kollegen in der Aviso-Debatte aufgefordert, sich „an die eigene Nase“ zu fassen und „unser eigenes Gutachterverhalten“ zu überprüfen. Darüber sollte die deutschsprachige Kommunikationswissenschaft reden, nachdem sich Peer Review hier etabliert hat.

\section{Literatur}

Baum, A., \& Schmidt, S. J. (Hrsg.). (2002). Fakten und Fiktionen. Über den Umgang mit Medienwirklichkeiten. Konstanz: UVK.

Bornmann, L. (2007). Peer-Review in der Wissenschaft - eine Analyse des Begutachtungsverfahrens aus der Sicht wissenschaftssoziologischer Theorien. Swiss Journal of Socio$\log y, 33,327-347$.

Bourdieu, P. (1998). Vom Gebrauch der Wissenschaft. Für eine klinische Soziologie des wissenschaftlichen Feldes. Konstanz: UVK. 
Ceci, S. J., \& Peters, D. (1984). How blind is blind review? American Psychologist, 39, 1491-1494.

Cicchetti, D. V. (1991). The reliability of peer review for manuscript and grant submissions: A cross-disciplinary investigation. Behavioral and Brain Sciences, 14, 119-135.

Debatin, B., \& Rath, M. (2014/15). Epilog: Moralität und Kollegialität. Aviso. Informationsdienst der Deutschen Gesellschaft für Publizistik- und Kommunikationswissenschaft, Nr. 59, 9.

Echterbruch, J., Lassen, J., \& Tholen, J. (2016). Von pragmatischen Post-Docs und progressiven Praktikern. Befunde einer DGPuK-Mitgliederbefragung zu Fachzeitschriften der Kommunikationswissenschaft. Publizistik, 61, 17-40.

Eysenck, H. J., \& Eysenck, S. B. G. (1992). Peer review: Advice to referees and contributors. Personality and Individual Differences, 13, 393-399.

Gerhards, J. (2002). Reputation in der deutschen Soziologie - zwei getrennte Welten. Soziologie, 19-33.

Gould, T. H. P. (2012). The future of peer review: Four possible options to nothingness. Publishing Research Quarterly, 28, 285-293.

Hanitzsch, T. (2015). Celebrating 25 years of Communication Theory: Growing diversity under heavy strain. Communication Theory, 25, 349-355.

Hanitzsch, T. (2016). Impact und Normalwissenschaft. Die Fachzeitschrift als Treiber einer neuen Wissenschaftskultur. Publizistik, 61, 41-50.

Hirschauer, S. (2004). Peer Review Verfahren auf dem Prüfstand. Zum Soziologiedefizit der Wissenschaftsevaluation. Zeitschrift für Soziologie, 33, 62-83.

Krotz, F. (2014/15). Vom Missbrauch einer guten Idee. Aviso. Informationsdienst der Deutschen Gesellschaft für Publizistik- und Kommunikationswissenschaft, Nr. 59, 7.

Langenbucher, W. R. (2016). Die „Philosophie“ der Herausgeberzeitschrift und ihr (notwendiger?) Wandel. Publizistik, 61, 7-15.

Lünenborg, M. (2014/15). Verzerrungseffekte im Peer-Review. Aviso. Informationsdienst der Deutschen Gesellschaft für Publizistik-und Kommunikationswissenschaft, Nr. 59, 6.

Magin, M., \& Engesser, S. (2014/15). Vom Nutzen und Nachteil des Peer-Review aus Sicht des Nachwuchses. Aviso. Informationsdienst der Deutschen Gesellschaft für Publizistik- und Kommunikationswissenschaft, Nr. 59, 4.

Merton, R. K. (1973). The sociology of science: Theoretical and empirical investigations. Chicago: University of Chicago Press.

Meyen, M. (2004). Wer wird Professor für Kommunikationswissenschaft und Journalistik? Ein Beitrag zur Entwicklung einer Wissenschaftsdisziplin in Deutschland. Publizistik, 49, 194-206.

Neidhardt, F. (1986). Kollegialität und Kontrolle - Am Beispiel der Gutachter der Deutschen Forschungsgemeinschaft (DFG). Kölner Zeitschrift für Soziologie und Sozialpsychologie, 38, 3-12.

Neuman, W. R., Davidson, R., Joo, S.-H., Park, Y. J., \& Williams, A. E. (2008). The seven deadly sins of communication research. Journal of Communication, 58, 220-237.

Robson, K., Pitt, L., \& West, D. C. (2015). Navigating the peer-review process: Reviewers' suggestions for a manuscript. Factors considered before a paper is accepted or rejected for the Journal of Advertising Research. Journal of Advertising Research, March, 9-17.

Ross, P. F. (1980). The sciences' self-management: Manuscript refereeing, peer review, and goals in science. Massachusetts: The Ross Company, Todd Pond. 
Scherer, H. (2014/15). Von Opfern und Tätern. Aviso. Informationsdienst der Deutschen Gesellschaft für Publizistik- und Kommunikationswissenschaft, Nr. 59, 4.

Schmidt, C. (2008). Analyse von Leitfadeninterviews. In U. Flick, E. von Kardoff, \& I. Steinke (Hrsg.), Qualitative Forschung. Ein Handbuch (S. 447-456). Reinbek: Rowohlt.

Smith, D. A. (2014). Some thoughts on sociology journal publishing in the 21st century. American Sociologist, 45, 197-202.

Smith, R. (2006). Peer review: A flawed process at the heart of science and journals. Journal of the Royal Society of Medicine, 99, 178-182

Vesnic-Alujevic, L. (2012). Peer review and scientific publishing in times of Web 2.0. Publishing Research Quarterly, 30, 39-49.

Weingart, P. (2001). Die Stunde der Wahrheit? Zum Verhältnis der Wissenschaft zu Politik, Wirtschaft und Medien in der Wissensgesellschaft. Weilerswist: Velbrück.

Wennerås, C., \& Wold, A. (1997). Nepotism and sexism in peer-review. Nature, 387, 6631, 341-343. 